\title{
A Class of Public Opinion Dissemination Model considering the Information Screening Mechanism
}

\author{
Suyalatu Dong \\ School of Computer Information Management, Inner Mongolia University of Finance and Economics, Hohhot 010070, China \\ Correspondence should be addressed to Suyalatu Dong; srguleng_1983@163.com
}

Received 31 August 2021; Accepted 12 November 2021; Published 27 November 2021

Academic Editor: Joni Zhong

Copyright (c) 2021 Suyalatu Dong. This is an open access article distributed under the Creative Commons Attribution License, which permits unrestricted use, distribution, and reproduction in any medium, provided the original work is properly cited.

\begin{abstract}
This paper introduces an information screening mechanism based on the general SEIR model of public opinion dissemination in complex networks. Specifically, networks users screen the information disseminated in complex networks based on their knowledge reserve, life experience, and personal preferences. This screening mechanism filters the public opinion disseminated. Based on the quantitative analysis of this mechanism, we establish a new SEIR model of public opinion dissemination in complex networks. The simulation results on the Facebook network data set provide theoretical guidance for the formulation of effective public opinion suppression strategies.
\end{abstract}

\section{Introduction}

With the rapid development of online social networks, the Internet has become more and more embedded in all aspects of people's lives. The development of computer technology has enabled proliferation of the social networks. According to the Global Digital Report published by We Are Social [1], the number of Internet users worldwide has exceeded 4 billion mark, confirming that around half of the world's population has, in some ways, "touched the Internet." According to the above report, the number of new Internet users increased by 250 million in 2017, of which the growth in Africa was dominant. The annual growth rate in Africa is 20 percent higher than that of Europe. In addition, driven by the widespread availability of smart phones and mobile data services, new mobile Internet users account for a larger proportion of Internet users. For instance, in 2017, more than 200 million people received their first ever mobile phones. In 2017, global social media usage grew by $13 \%$, bringing the total number of users to 3.196 billion [1].

In China, although the popularity of the Internet lagged more than that of the developed countries in Europe and America, the development of online social networks has been very rapid. Instances such as popularity of the instant messaging social software, Wechat with more than 1.08 billion active users in 2018, and phenomenal increase in the users of short video social software-TikTok, with more than 426 million active users in 2018, indicate a rather strong momentum of domestic Internet development in China.

Recently, China's Xi'an Benz oil spillage has spread through and became a trend in social media such as TikTok and Sina Weibo. The story was widely forwarded across and took tens of millions of comments in social media and quickly became a hot topic on the Internet. Such incidents corroborate on the power of public opinion dissemination on the popular online social networks.

The largest Chinese Journal Database Website in China, Howmet [2], has indexed 84 Chinese Journal Papers in the past five years on the topic "public opinion dissemination in complex networks." This number continues to increase year by year. Therefore, this is evident that the study of public opinion dissemination in complex networks is amongst the hot research fields at home and abroad. This research area concerns with qualitative and quantitative research on the way public opinion is disseminated in complex social networks. The research method often includes establishing a dynamic differential equation of public opinion dissemination which then facilitates simulating the process of negative public opinion dissemination on the data set of social networks. This helps making prediction on the trend 
of negative public opinion dissemination and further provides insights on the mechanism of negative public opinion dissemination. The results are then utilized to propose efficient strategies to suppress the outbreak of negative information.

For quantitative analysis, domestic researchers often adopt methods based on SIS compartment model [3], SIR compartment model [4], and their corresponding evolution models. They then carry out numerical simulation and simulation experiments on the mechanism of public opinion disseminations. In this process, scholars at home and abroad have constructed a variety of public opinion dissemination models; see, e.g., [5-19]. These research works focus on the actual network topology and the characteristics of public opinion dissemination in the network. Nevertheless, such models ignore an important issue; that is, the information itself has the attribute of knowledge. Chen [20] used the improved SEIR model to establish the WeChat public opinion dissemination model that considers the group effect and the microblog public opinion dissemination model that considers the influence of users and uses a two-layer coupling network to describe the cross-media dissemination process of public opinion. Yin et al. [21] established an improved SEIR model based on the characteristics of infectious disease transmission and discussed that the transmission probability is not a constant. It is a variable determined by the restriction of social relations, controlled by external factors and the overlapping spread of news in "small society." Fan et al. [22] considered the assumption that the model's assumption of equal propagation probability between network nodes per unit time is inconsistent with the nature of the actual social network's propagation as well as the fact that immune nodes have a certain inhibitory effect on the spread of rumors in actual situations and proposed an improved rumor spread model PSEIR. Zhu et al. [23] introduced influencing factors such as user similarity, information value, and information timeliness to construct an improved SEIR public opinion dissemination model suitable for explaining the law of public opinion dissemination in WeChat Moments. Li et al. [24] defined the user influence, topic popularity, topic interest to analyze the process of opinions fusion among the users under the interest and confidence threshold and proposed a new public opinion evolution HK-SEIR model which combines the opinion fusion $\mathrm{HK}$ and the epidemic transmission SEIR models. Zhang et al. proposed [25], according to the individual state division method of compartment model, a network public opinion propagation model which is constructed based on user interest matching through state transition analysis and inference of state transition probability. Jiang et al. [26] proposed a two-stage rumor model, namely, susceptible, positive, negative, and recovered state (SPNR), which is built to analyze rumor spread and reversal of rumors regarding emergencies on Weibo. The model considers the hysteresis of official news and public swing mentality based on epidemic models, selected to analyze the effect of influential factors in different dimensions on the dissemination of social media public opinion information using econometric models. These models do not consider the time-varying characteristics of the infection rate, recovery rate, transfer rate, and other parameters in the propagation process and directly select these parameters as a fixed constant. And the model does not consider the inactive characteristics of immune nodes, latent nodes, propagation nodes, and immune nodes in the process of rumor propagation. That is, it is not considered that various nodes in the network have a natural exit rate in the process of rumor propagation. And the model does not consider the inactive characteristics of immune nodes, latent nodes, propagation nodes, and immune nodes in the process of rumor propagation. That is, it is not considered that various nodes in the network have a natural exit rate in the process of rumor propagation. Moreover, in this model, we introduce the natural exit rate and consider the inactive nature of various types of nodes in the process of rumor propagation. Furthermore, this model uses the multiagent simulation modeling software NetLogo for simulation modeling, which simulates the individual behavior mode and the interaction process between individuals.

When information is disseminated on complex networks, instead of just passively accepting the information, users screen the information from the perspective of their own knowledge base, life experience, and even personal preferences. A common issue in most information propagation models in complex networks is that the healthy nodes will be changed into dissemination nodes or latent nodes with a certain probability. This implicitly ignores the underlying users' screening mechanism which filters out false or uninteresting information.

A recent trend is to investigate the information communication model on multilayer coupling network. Nevertheless, the current research on information transmission model in multilayer coupling networks often overlooks the following issues: (1) how to determine which layer of the information transmission plays a leading role. This is essential as, in the multilayer coupling network, the information transmission rate on each layer might not be equal. (2) The second issue is how to determine which node in that layer is the key node in the process of public opinion dissemination in multilayer coupling network. Note that the number and degree of nodes in each layer of multilayer coupling network might be different. (3) Most of the multilayer coupling network public opinion dissemination models are based on the assumption that the public opinion dissemination process between different levels has a promoting effect on each other. This might not be the case, as the layers might be able to affect/restrict each other. The above-mentioned issues need to be further discussed; therefore, we focus our research on the public opinion dissemination process in the single-layer networks.

In the process of establishing the model, this paper considers users' information screening mechanism. In our research, we assume that due to the influence of users' knowledge base, life experience, and personal preferences, when they first come across new information, they will filter out part of the information that they are not interested in or believe to be false. 


\section{Modeling}

The SEIR model of public opinion dissemination in complex networks with information screening mechanism divides nodes into four categories: (1) when the information flows into the Facebook network, the node users who have access to information will become healthy nodes $S$. (2) The healthy nodes $S$ that have obtained the information will judge the information according to their own preferences and information content. Some healthy nodes $S$ will choose to hold a reserved attitude to this information, thus turning into a latent node $E$. therefore, the healthy nodes $S$ with $\alpha$ probability will be transformed into latent nodes $E$. In this case, the nodes are in a state of no cognition; some healthy nodes choose to ignore these information directly because they have certain life experience, knowledge reserves, and scientific literacy, and the healthy nodes $S$ with $\xi$ probability are directly transformed into immune nodes $R$. Some healthy nodes $S$ will choose to believe these information unconditionally due to lack of life experience, insufficient knowledge reserve, and low scientific literacy, so they will change into dissemination nodes $I$. Therefore, healthy nodes $S$ with $\varepsilon$ probability will change to dissemination nodes I. (3) After reading the information, the latent node $E$ comprehensively considers whether to implement the public opinion dissemination behavior. Therefore, the latent nodes $E$ with $\beta$ probability will choose to implement the public opinion dissemination behavior and change into dissemination nodes $I$. At the same time, the latent nodes $E$ with $\sigma$ probability will choose to directly transform into immune node $R$. (4) The dissemination nodes $I$ can choose to directly transform into immune node $R$, after implementing the dissemination behavior. Therefore, the dissemination nodes $I$ with $\gamma$ probability will directly change to immune nodes $R$. (5) Due to the derivative nature of topic-based communication, topics will generate new information and flow into Facebook network in different forms. Therefore, users who have become immune nodes $R$ may browse the latest news of the topic and turn into healthy nodes $S$. Therefore, the immune nodes $R$ with $\varphi$ probability will be transformed into healthy nodes $S$ again. The mechanism of public opinion dissemination is shown in Figure 1. The dynamic differential equation of continuous SEIR model with information screening mechanism is given by equation (1).

$$
\left\{\begin{array}{l}
S_{\prime}(t)=-\alpha S(t) I(t)-\varepsilon S(t) I(t)-\xi S(t) I(t)+\varphi R(t), \\
E_{\prime}(t)=\alpha S(t) I(t)-(\beta+\sigma+d) E(t), \\
I^{\prime}(t)=\varepsilon S(t) I(t)+\beta E(t)-(\gamma+d) I(t), \\
R_{\prime}(t)=\xi S(t) I(t)+\gamma I(t)+\sigma E(t)-(\varphi+d) R(t) .
\end{array}\right.
$$

In particular, it should be pointed out that the first equation of (1) is $-\alpha S(t) I(t)$ rather than $-\alpha S(t) E(t)$, because the healthy nodes $S$ can only program the latent nodes $E$ after contacting the dissemination nodes $I$. In (1), $S(t)$ is the proportion of healthy nodes in the network at time $t$; $E(t)$ is the proportion of latent nodes at time $t ; I(t)$ is the

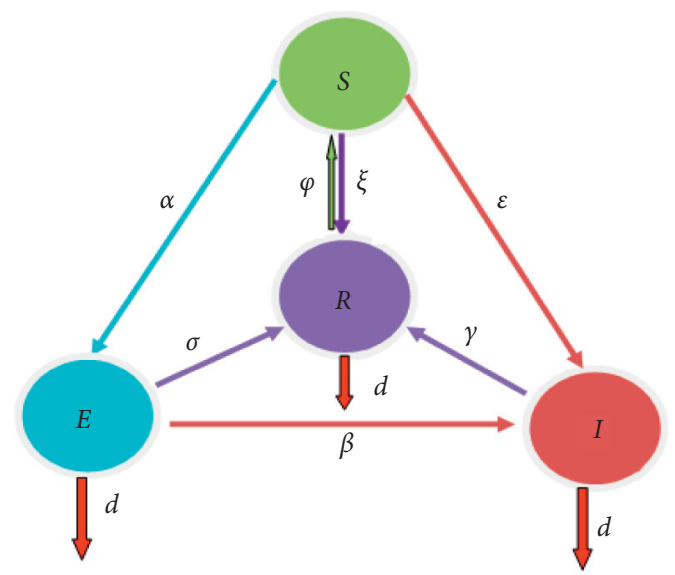

FIGURE 1: SEIR model of public opinion dissemination in complex network with information screening mechanism.

proportion of dissemination nodes at time $t$ in the network; $R(t)$ is the proportion of immune nodes at time $t$ in the network. Note that although there is a natural roll out rate, $d$, in the process of public opinion dissemination, as $d$ is very small, we can write $S(t)+E(t)+I(t)+R(t) \approx 1 . \alpha, \varepsilon, \xi, \varphi, \beta$, $\sigma, \gamma$ represent the probability of conversion between nodes, $0<\alpha, \varepsilon, \xi, \varphi, \beta, \sigma, \gamma<1$.

We quantify the user's screening mechanism using two sets of coefficients. The first group of coefficients are $\varepsilon$ and $\xi$, which means that when users are new to the information, they will screen the information using their personal preferences, knowledge base, life experience, scientific literacy, and other factors or filter the information out that is not interesting or deems false. That is to say, they will become immune nodes or they will develop a strong trust in the information. Information or judging the authenticity of information becomes the forwarder of information, and thus they make positive comments; that is to say, they become a dissemination node. The second group of coefficients include $\sigma$, which means that after receiving the information, the user is unable to judge the credibility of the information due to the limitations of their knowledge base and life experience or maintain a neutral attitude towards the information and put it on hold for the time being. However, with the continuous emergence of similar information or evolutionary versions of this kind of information, the information will be judged accordingly and then filtered out as uninterested or untrue information.

Reference [27] quantitatively describes the effect of social reinforcement on public opinion dissemination process. Social reinforcement refers to the praise and negation of individual behavior by society. As a result of social reinforcement, the individual's instrumental response tendency is enhanced, and the individual forms or changes some behavior habit or behavior tendency. It can be seen that the first group of coefficients $\varepsilon$ and $\xi$ proposed in this paper are similar to the quantitative description of social reinforcement mechanism, information receiving probability $\lambda$, discussed in reference [27]. Specifically, with the increase of users with less knowledge reserve, less scientific literacy and less profound life accumulation, individual users will be 
affected to make positive reactions to negative public opinion (that is, praise, comment, or forward the information). In this paper, $\varepsilon$ is used to describe it. With the increase of users with rich knowledge reserve, high scientific literacy, and deep life accumulation, individual users will be affected to make negative reactions to negative public opinion (that is, individuals become immune nodes). In this paper, $\xi$ is used to describe it. The second group of coefficients $\sigma$ proposed in this paper for public opinion screening mechanism are exactly the same as that of information receiving probability $\lambda$, which is the quantitative description of social strong social reinforcement mechanism discussed in reference [27], because in the process of proposing $\sigma$, memory and cumulative effects of public opinion dissemination are also considered. In conclusion, according to the formula of information receiving probability $\lambda$ in reference [27], the quantitative descriptions of the three coefficients $\varepsilon$, $\xi$, and $\sigma$ of the screening mechanism are as follows:

$$
\left\{\begin{array}{l}
\varepsilon=\frac{1}{1+\psi_{1} e^{-b_{1}\left(\omega_{1}-1\right)},} \\
\xi=\frac{1}{1+\psi_{2} e^{-b_{2}\left(\omega_{2}-1\right)}}, \\
\sigma=\frac{1}{1+\psi_{3} e^{-b_{3}\left(\sum \omega_{i j}-1\right)}},
\end{array}\right.
$$

and among them, $\omega_{1}$ and $\omega_{2}$ respectively indicate the ratio of the number of users with insufficient knowledge accumulation and low scientific literacy to those with rich knowledge accumulation and high scientific literacy, and the ratio between the number of users with rich knowledge accumulation and high scientific literacy and the number of users with insufficient knowledge accumulation and low scientific literacy; $\omega_{1}$ and $\omega_{2}$ are reciprocal. What needs to be further explained is that the setting of $\omega_{1}$ and $\omega_{2}$ has the following considerations. Here we take $\omega_{2}$ as an example, if the number of users with rich knowledge accumulation and high scientific literacy is greater than the number of users with insufficient knowledge accumulation and low scientific literacy, $\omega_{2}$ will be greater than 1. If $\omega_{2}$ is greater than 1 , then $b_{2}$ will increase, and the corresponding $\xi$ will increase. The increase of $\xi$ means that the user's resistance to negative information is higher, which is consistent with the original intention of setting $\xi$, the same meaning of $\omega_{1}$ is similar; $\sum \omega_{i j}$ is a parameter that takes into account the memory and cumulative effects of individual users' public opinion dissemination; $\psi_{1}, \psi_{2}$, and $\psi_{3}$ are adjustable parameters. $1 / 1+\psi_{1}, 1 / 1+\psi_{2}$ and $1 / 1+\psi_{3}$ are similar to the propagation rate, the increase of $\psi_{1}, \psi_{2}$, and $\psi_{3}$ will lead to the decrease of their corresponding functions $\varepsilon, \xi$, and $\sigma$. Parameters $b_{1}, b_{2}$, and $b_{3}$ can be called reinforcing factors. When conditions $\omega_{1}-1>0, \omega_{2}-1>0$, and $\sum \omega_{i j}-1>0$ are satisfied, the increase of parameters $b_{1}, b_{2}$, and $b_{3}$ will increase their corresponding functions $\varepsilon, \xi$, and $\sigma$, and vice versa.
In order to study, in the following discussion of this paper, $\varepsilon, \xi$, and $\sigma$ are taken as definite constants. The impact of the quantitative description of $\varepsilon, \xi$, and $\sigma$ in the process of public opinion dissemination will be discussed in our future work.

In the third part, we analyze the numerical simulation results of the discrete SEIR model (3); in the fourth part, we analyze the simulation results of the continuous SEIR model (1) and compare the two models.

\section{Numerical Simulations of the Discrete SEIR Model}

In the process of numerical simulation, because it is a pure numerical calculation, no underlying graph is used, and because the natural roll-out rate $d$ is very small, it is assumed that the total population $N$ is almost constant in the numerical simulation process. In the process of numerical simulation, the parameter selection is shown in Table 1. Figure 2 shows the numerical simulation results of the discrete SEIR model. Figure 2 indicates that for $t>10$ the density of dissemination nodes $I(t)$ increases and reaches its highest peak around $t=40$. This indicates that the public opinion has the greatest influence among online social network users. It is also seen that the density of dissemination nodes $I(t)=0$ at $t=100$. This means that the public opinion fever is gradually decreasing until it completely disappears. The attenuation of healthy nodes $S$ ( $t$ ) density also begins at the time of $t=10$, which coincides with the gradual increase of dissemination nodes density at $t=10$. For times around $t=60$, the density of healthy nodes reaches to the lowest value, and then $S(t)=0$. This indicates that all healthy nodes in the network have been exposed to the public opinion. Furthermore, we notice that the peak value of the curve for the latent node density $E(t)$ varying with time in numerical simulation is much smaller than that of the curve of dissemination nodes density $I(t)$. This situation is different from the numerical simulation results of the public opinion dissemination model presented by previous researchers. This is because we consider the user filtering mechanism in the improved SEIR model. The screening mechanism of public opinion is as follows. When a healthy node contacts a public opinion in the network, the healthy node first judges the truth of the public opinion according to its personal preferences, knowledge base, and life experience. The user then chooses whether to praise, forward, and comment on the public opinion from the positive or negative perspective. Healthy nodes are less likely to be depressed, which is also in line with the reality of social network users' treatment of public opinion. Of course, one cannot ignore the latent nodes when discussing the choices that users make upon; they first come across the public opinion in social networks.

It can also be seen from Figure 2 that the immune node density $R(t)$ increases gradually with time for $t>10$. For $t=100$, the immune node density $R(t)$ reaches its maximum, and then the density of the other three nodes becomes zero. This indicates that the influence of public opinion in social 
TABLE 1: The default system parameters.

\begin{tabular}{lcc}
\hline Parameter & Parameter description & Value \\
\hline$N$ & Initial total population & 6392 \\
$S_{0}$ & Initial healthy nodes & 6388 \\
$I_{0}$ & Initial dissemination nodes & 4 \\
$E_{0}$ & Initial latent nodes & 0 \\
$R_{0}$ & Initial immune nodes & 0 \\
$\xi$ & Probability of healthy node transforming into immune node & 0.0200 \\
$d$ & Natural exit rate & 0.0005 \\
$\sigma$ & Probability of latent node transforming into immune node \\
$\varphi$ & Probability of immune node transforming into healthy node & 0.0250 \\
$\gamma$ & Recovery rate & 0.0060 \\
$\alpha$ & Probability of healthy node transforming into latent node & 0.0680 \\
$\varepsilon$ & Infection rate & 0.2600 \\
$\beta$ & Probability of immune node transforming into dissemination node & 0.0050 \\
\hline
\end{tabular}

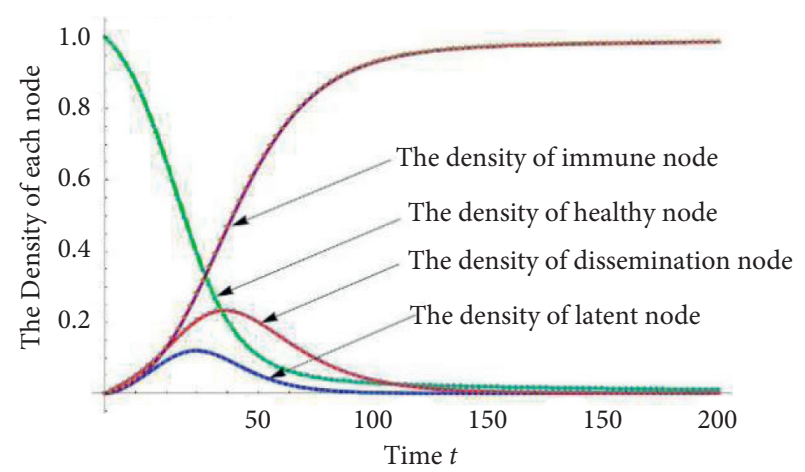

Figure 2: The numerical simulation of model.

network completely disappears for $t>100$ and all healthy nodes will adopt the public opinion.

In the foregoing, we have quantified the screening mechanism into two groups with three parameters. Figures 3-5 show the effect of three parameters on the public opinion dissemination when they are different. Figure 3 shows the time-varying curve of the dissemination nodes density when other parameters are fixed and $\varepsilon$ is $0.001,0.005$, 0.009 , and 0.013 , respectively. As it is seen in Figure 3, with the increase of $\varepsilon$, the density of dissemination nodes changes with time, and the peak time of the curve will be ahead of time and the peak value will increase. This shows that the transformation rate of healthy nodes into dissemination nodes $\varepsilon$ improves the role of the peak time and peak value of dissemination node density with time.

Figure 4 shows the time-varying curves of the density of dissemination nodes for $\sigma$ is $0.025,0.045,0.065$, and 0.085 , respectively, where other parameters remain unchanged. From Figure 4, it can be seen that by increasing $\sigma$, the peak value of the curve of dissemination node density changes with time with a lag behind, and the peak value also decreases. It is shows that the conversion rate from latent node to immune node has a repressive role for the peak time and peak value of dissemination node density with time.

Figure 5 shows the time-varying curve of the node density where the other parameters are unchanged and for $\xi$ it equals $0.002,0.006,0.010$, and 0.014 , respectively. Figure 5 shows that the transformation rate from healthy node to

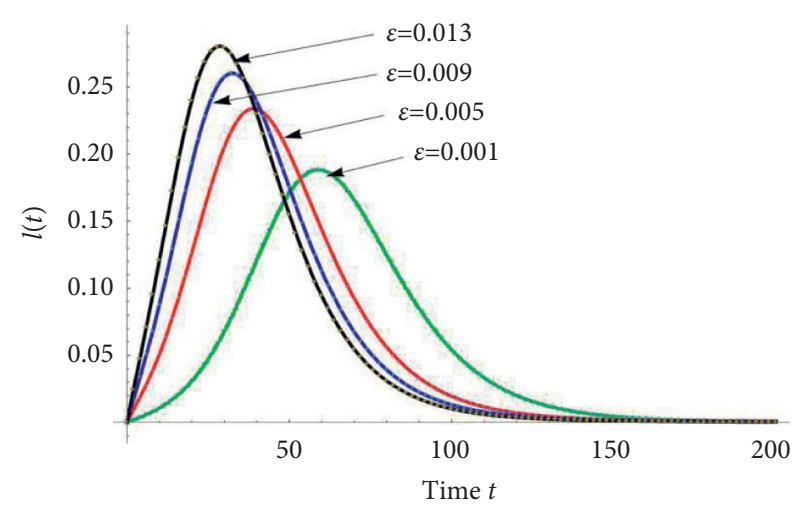

FIgURE 3: The change of public opinion dissemination scale with time when $\varepsilon$ takes different values.

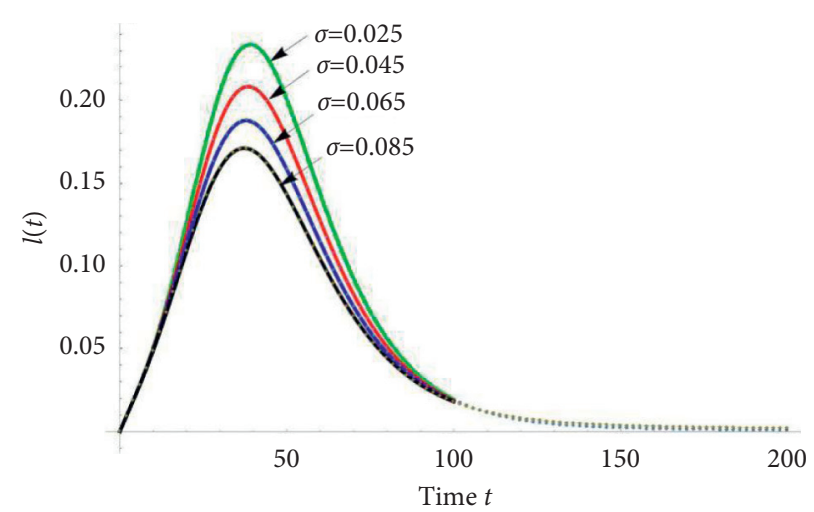

Figure 4: The change of public opinion dissemination scale with time when $\sigma$ takes different values.

immune node $\xi$ repressive role for the peak time and peak value of dissemination node density with time. In our modeling regard $\xi$ is a parameter that quantifies the screening mechanism and further measures the influence on the public opinion dissemination.

The above results show that amongst three coefficients which quantify the user's screening mechanism, the influence of $\varepsilon$ on public opinion dissemination is dominant while the effect of the two other parameters, $\xi$ and $\sigma$, is relatively weak. 


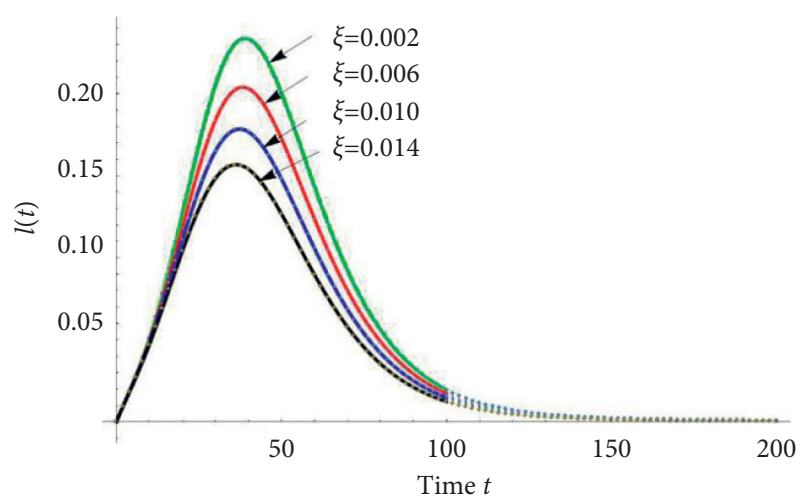

FIGURE 5: The change of public opinion dissemination scale with time when $\xi$ takes different values.

\section{Simulation Experiment of the Continuous SEIR Model}

Before introducing the simulation experiment, we will focus on the difference and emphasis between numerical simulation and simulation experiment. The fourth section is the part of numerical simulation. We use the scientific computing software Mathematica to get the solution of discrete propagation model (i.e., discrete differential equations) and then make numerical analysis. The specific method is to bring the parameter values of all given parameters into the discrete differential equation, set the range of independent variable $t$ (such as $0-100$ ), and then get the numerical solution of discrete differential equations through Mathematica. On this basis, a series of mathematical analysis is carried out, such as changing a specific parameter under the condition that other parameters remain unchanged, so as to analyze how the change of parameter value affects the value of numerical solution of discrete differential equations. The fifth part is the simulation part. We use the multi-agent modeling and simulation platform to simulate the continuous propagation model. The specific method is as follows: first, search for the rumor spreading sample model in the NetLogo sample library, and the specific path is Sample ->Social Science->Rumor Mill; then, read the data from Facebook into the NetLogo . At this time, each node in the Facebook network can be regarded as an agent; then, we rewrite the rumor mill sample model provided by NetLogo according to the propagation rules defined in the continuous propagation model, that is, select the agent behavior based on the propagation rules defined in the continuous propagation model, and agents with adjacency or path connection will influence each other's behavior. That is to say, the network topology is also one of the factors to be considered in the simulation experiment. It can be seen from the above analysis that the simulation experiment in Section 5 is to verify whether the continuous propagation model can better reflect the actual situation of public opinion dissemination in the real world, which is an approximate simulation of the real world.

The following describes the simulation experiment process.
4.1. Data Set Description. We simulate the model on the Facebook network data set provided in [28]. The Facebook network data set comprises of 6392 nodes and 217700 edges. A visualization of the simulated network is shown in Figure 6(a). As it is seen in the graph, the network is scattered outward from a node; therefore, the data set contains a list of friends of a user and further a list of friends of his/her friends in Facebook network. Figure 6(b) shows the degree distribution of the network. The graph also shows that the network presents a power-law distribution characteristic, indicating that the network is scale-free. The scalefree characteristic of the network indicates that it is possible for the network to have a high number of nodes. Therefore, the network is vulnerable to intentional attacks [29]. In Figure 6(c), the average clustering coefficient distribution of the network is shown. The average clustering coefficient of the network obtained by Gephi software tool is 0.247 . This shows that the network has an obvious module structure and the average distance between the nodes which is relatively small [30]. In Figure 6(d), A report on the distance between Facebook network nodes was analyzed on Gephi. Through the analysis, the average path length of Facebook network is 1.95. We generate a random network of the same scale and analyze the topology of the random network. As shown in Figure 7, the average path length of random network is 6.25 , while its average clustering coefficient is 0.04 . Through the analysis of the degree distribution characteristics, clustering coefficient and average path length, and other topological characteristics of the Facebook network and random network, it can be known that the selected data set in the simulation experiment of the continuous SEIR model has the characteristics of small world network.

4.2. Simulation Experiment. We simulated the continuous model using real Facebook social network data sets. In the process of simulation experiment, the parameter selection is shown in Table 1. Because the average degree of the network is 68.106, we select few nodes with degree 68 as the propagation node, $I_{0}$, and at the initial time we set $I_{0}=4$. Based on the above data set, we use NetLogo (a multiagent simulation platform) to realize the simulation experiment of the continuous SEIR model. The NetLogo simulation platform simulates the behavior of micro individuals, the emergence of macro models, and the relationship between them. Figure 8 shows the interface of NetLogo multiagent simulation platform. It is seen that the value of each parameter can be changed through the slider in NetLogo, which makes the simulation process more flexible. Figure 9 shows the simulation results of continuous SEIR model with screening mechanism. Compared with the numerical simulation results of discrete model in Figure 2, we observe that the peak time for the density of each node happens earlier in time and the peak value is also relatively larger. In the simulation experiment of continuous dissemination model, the propagation range is obviously expanded, which may be related to the consideration of network topology in simulation experiment. In the simulation experiment, when selecting the initial propagation nodes, the nodes with higher degree 


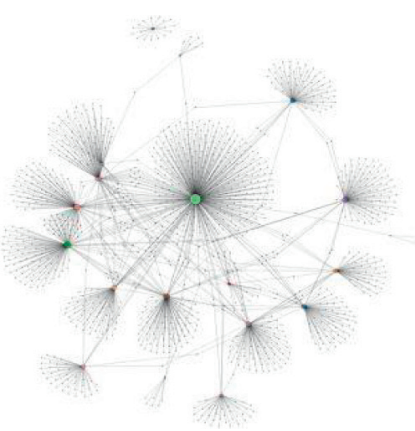

(a)

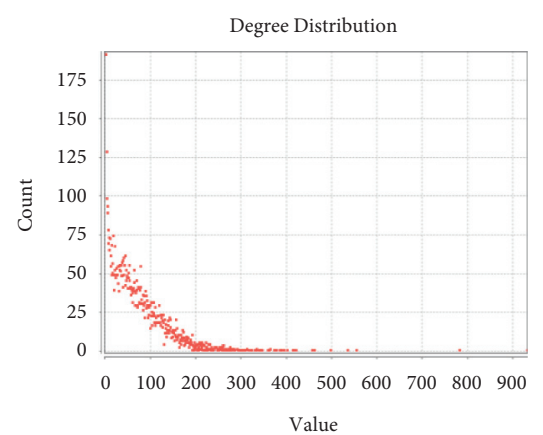

(b)

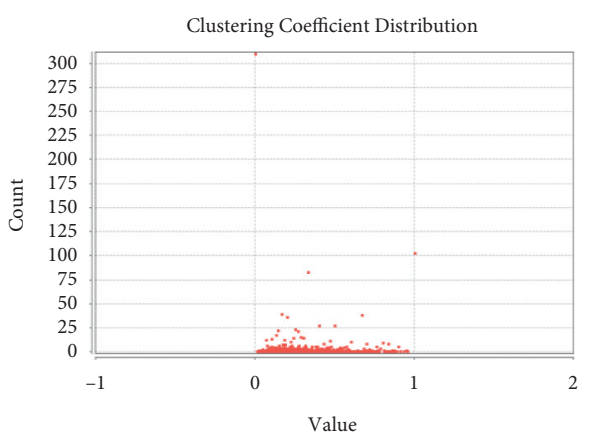

(c)

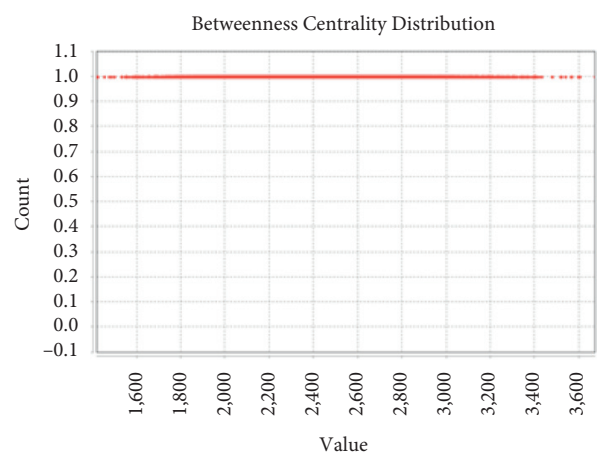

(d)

Figure 6: Facebook network topology. (a) Facebook network visualization. (b) Node degree distribution. (c) Average clustering coefficient distribution. (d) Betweenness centrality distribution.

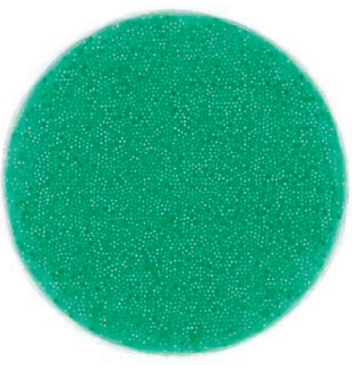

(a)

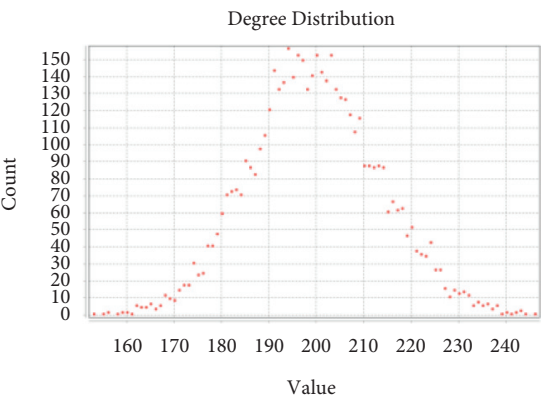

(b)

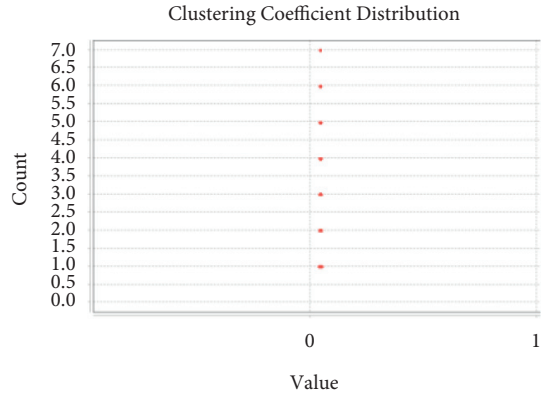

(c)

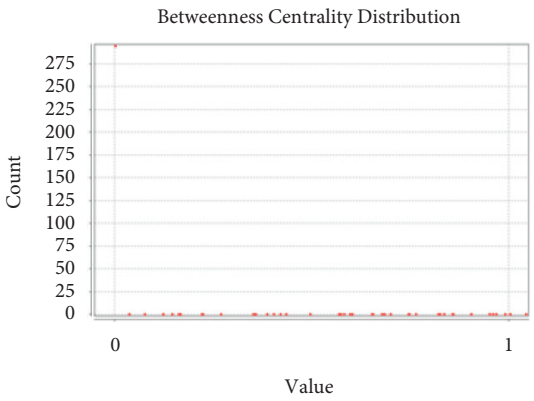

(d)

Figure 7: Random network topology. (a) Random network visualization. (b) Nodes degree distribution. (c) Average clustering coefficient distribution. (d) Betweenness centrality distribution.

in the network are selected. However, according to reference [31], the propagation influence of node's attribute (K-shell value) is much greater than that of node's local attribute (degree). In the simulation experiment, the propagation range is obviously enlarged, and the possible reason is that a certain initial propagation node with larger $\mathrm{K}$-shell value is 


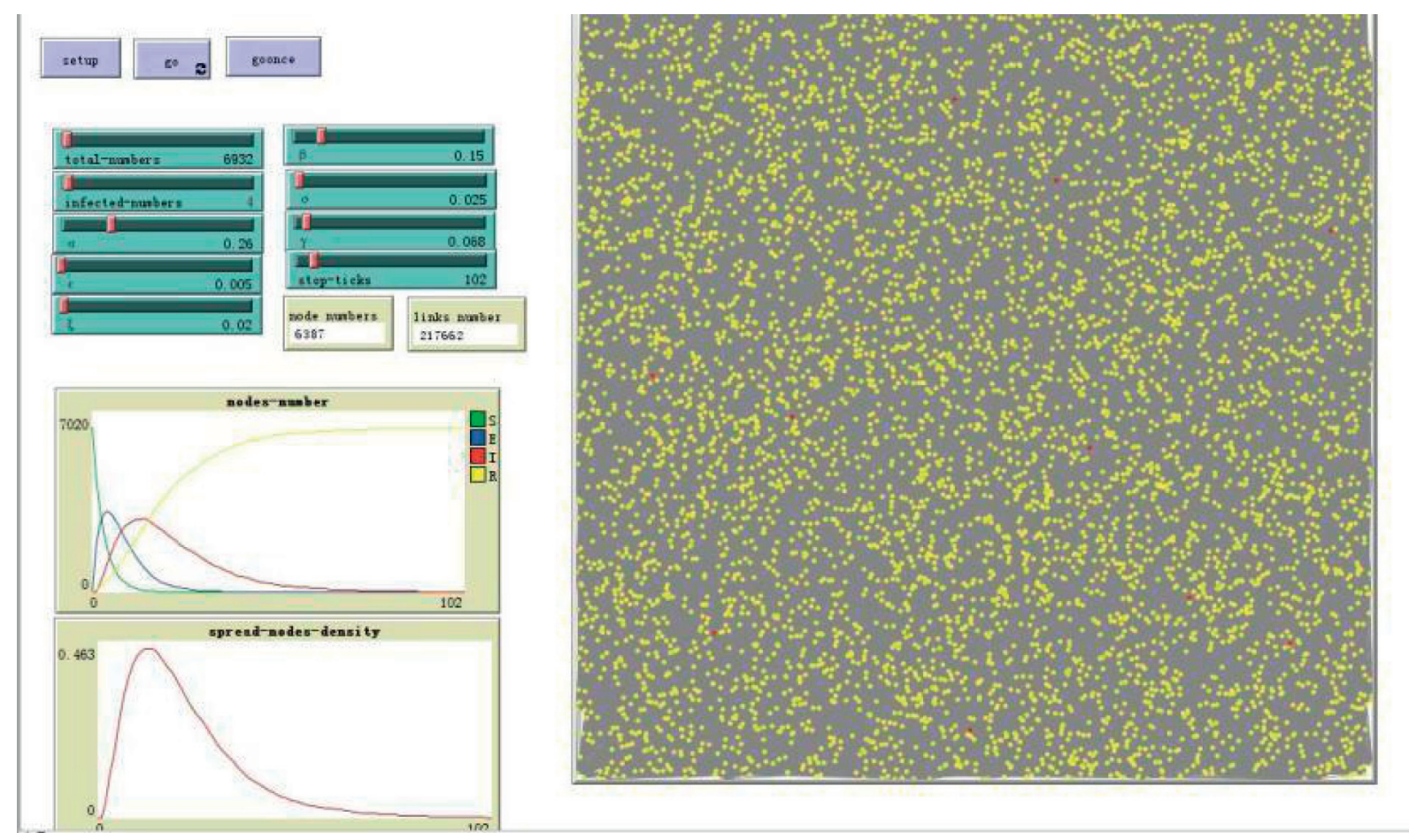

FIgURE 8: The simulation interface.

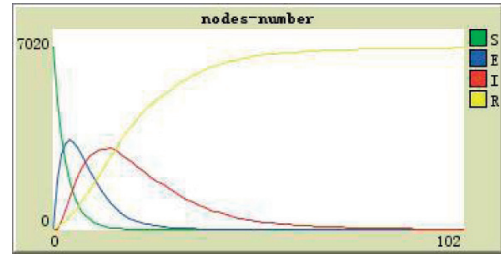

(a)

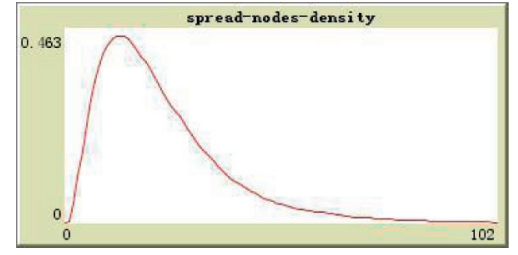

(b)

FIgURE 9: The simulation results of the continuous model.

selected. The influence of node $\mathrm{K}$-shell value on propagation influence will be further discussed in future research. It can also be seen from Figure 9 that the number of dissemination nodes can account for $46.3 \%$ of the total nodes which is the influence scope, or dissemination effect, of the public opinion. Figure 10 shows the dynamic visualization of the agents representing different types of nodes in different time periods. It can be seen in Figure 10 that at $t=25$, there are a large number of red-colored agents, indicating that at $t=25$, public opinion has had a greater impact on the public; i.e., almost $46.3 \%$ of the public believe in the authenticity of the public opinion, and this point of time is very important for public opinion control or public opinion guidance.

In the simulation experiment, we also analyze the impact of each parameter which represents the information screening mechanism on the public opinion dissemination. Figure 11 shows the change in the proportion of the number of dissemination nodes to the total number of nodes, where $\varepsilon$ takes different values during the simulation experiment. Figure 11 shows that with the increase of $\varepsilon$, the proportion of dissemination nodes to the total number nodes is also increasing. This provides further evidence of the significant importance of $\varepsilon$ on the spread of public opinion, which is consistent with that of numerical simulation of discrete model.

Figure 12 shows the change in the proportion of the number of dissemination nodes to the total number of nodes for different values of $\sigma$. It can be seen from Figure 12 that by increasing the value of $\sigma$ from $\sigma=0.025$ to $\sigma=0.040$, the proportion of the number of dissemination nodes to the total number of node is also increased. In contrast, by increasing the value of $\sigma$, from $\sigma=0.040$ to $\sigma=0.060$, the proportion of the number of dissemination nodes in the total number of nodes is decreased. This shows that a certain range of values for $\sigma$ promotes the spread of or hinder the spread of public opinion. Note that this result is not consistent with that of presented in the numerical simulation. The main reason is that the topology of the network is considered in the simulation experiment of the continuous model; that is, the nodes with degree of 68 are selected in the simulation experiment, while the numerical simulation only analyzes the model from the pure numerical point of view. However, whether the propagation influence comes from node degree or from node attribute (K-shell value) needs to be further studied in the future. 


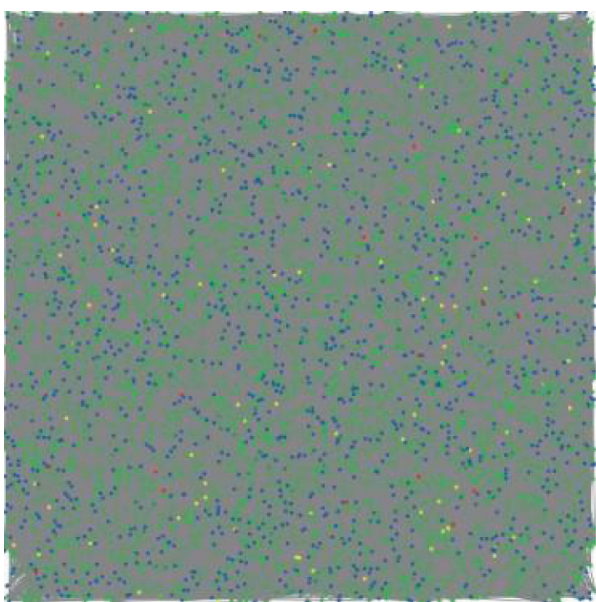

(a)

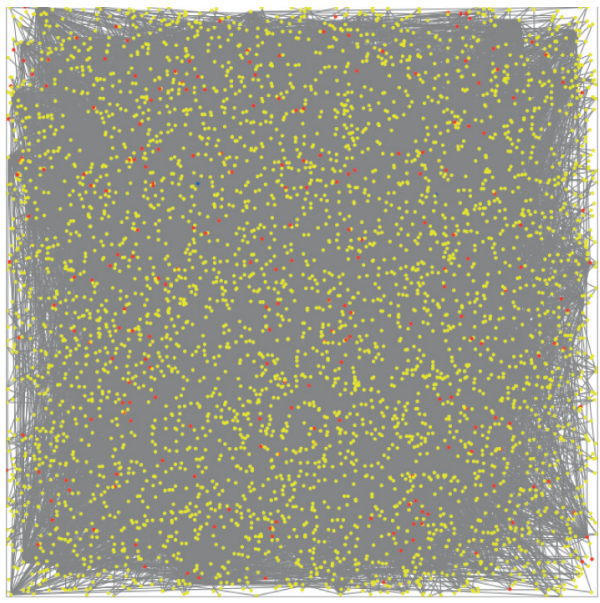

(c)

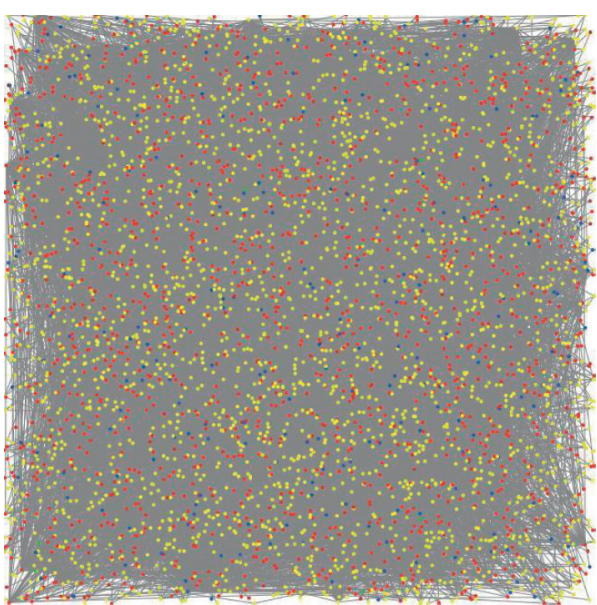

(b)

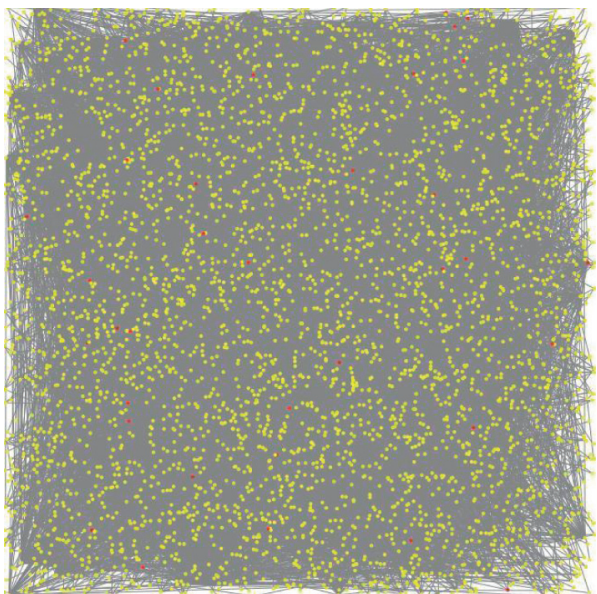

(d)

Figure 10: State of each node at different times. (a) $(t=5)$. (b) $(t=25)$. (c) $(t=50)$. (d) $(t=75)$ (red colors represent dissemination nodes).

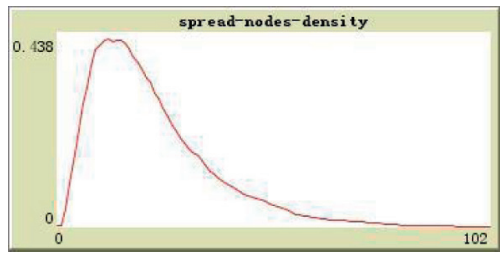

(a)

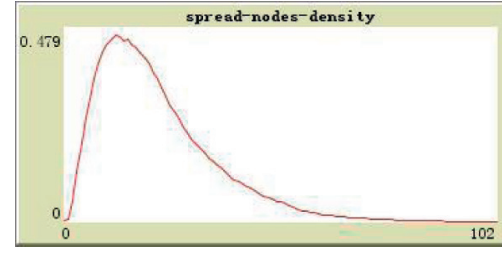

(b)

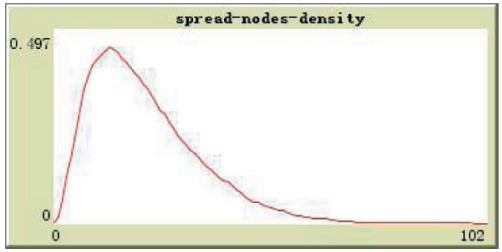

(c)

Figure 11: . The change of dissemination nodes density for different values of $\varepsilon(N=2000)$. (a) $\varepsilon=0.005$. (b) $\varepsilon=0.010$. (c) $\varepsilon=0.020$.

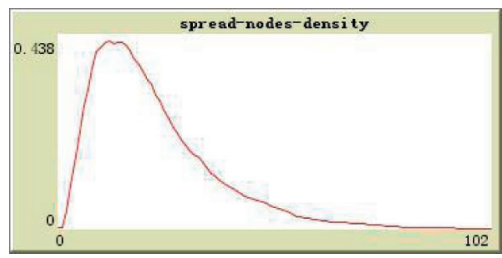

(a)

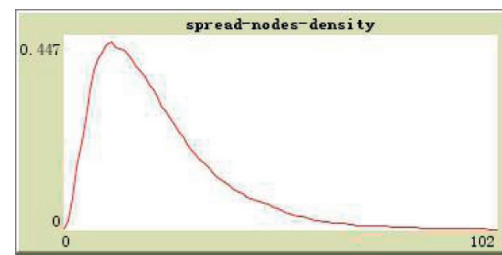

(b)

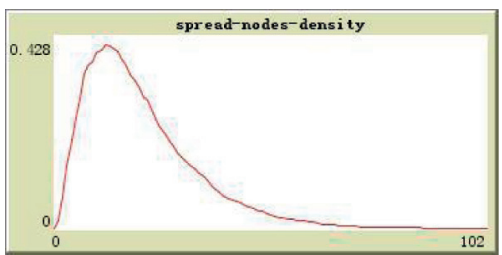

(c)

Figure 12: The change of dissemination nodes density for different values of $\sigma(N=2000)$. (a) $\sigma=0.025$. (b) $\sigma=0.040$. (c) $\sigma=0.060$. 


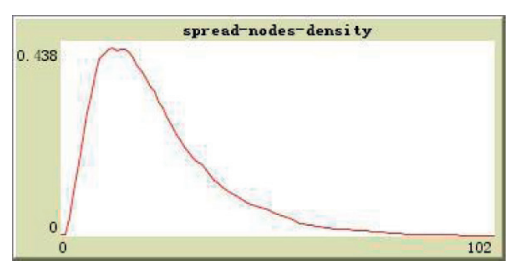

(a)

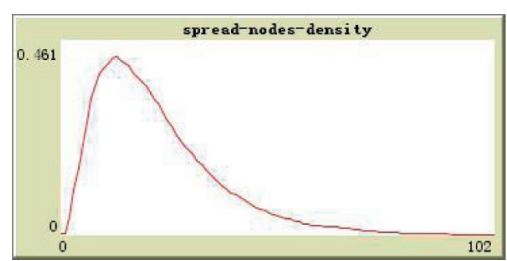

(b)

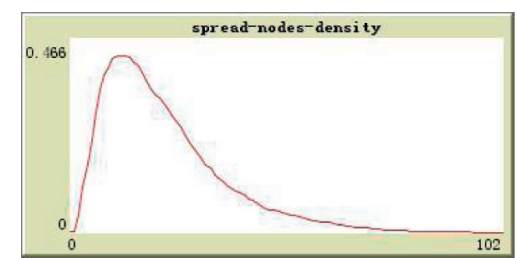

(c)

FIGURE 13: The change of dissemination nodes density for different values of $\xi(N=2000)$. (a) $\xi=0.020$. (b) $\xi=0.030$. (c) $\xi=0.040$.

Figure 13 shows the change of the proportion of the number of dissemination nodes in the total nodes for different values of $\xi$. Figure 13 shows that with the increase of $\xi$, the proportion of the number of dissemination nodes in the total number of nodes is also increasing. This further shows the leading influence of public opinion communication. It can also be seen that with the increase, this effect gradually weakened. This result is not consistent with the numerical simulation. This is because the topology of the network is considered in the simulation experiment, but not in the numerical simulation.

\section{Conclusions}

In the study of many public opinion dissemination models, researchers often assume that the healthy nodes are like a blank sheet of paper. In such models' healthy nodes when facing the public opinion, they have no preference and judgment ability; thus. they join in the process of public opinion dissemination unconditionally. However, the reality is that online social network users do not often accept public opinion. They have in fact a point of personal interest and make their own judgment whether they like to join in the dissemination of public opinion based on their life experience and knowledge base.

This paper established a public opinion dissemination model with screening mechanism. We noted that healthy nodes often consider whether they should participate in the process of public opinion dissemination from the perspective of their knowledge base, life experience, and personal preferences. In this process, users may directly judge the authenticity of the information contained in the public opinion and thus directly become immune nodes and do not participate in the process of public opinion dissemination. In our model, two groups of three parameters were used to quantify the screening mechanism, and further numerical simulation of discrete model and simulation experiments of continuous model were carried out. The numerical simulation results of continuous model showed that as a quantitative parameter of the screening mechanism, the influence on the process of public opinion propagation is not great, which is different from our original hypothesis. The simulation results of continuous model showed that due to the influence of network topology on the propagation process considered (as for the propagation influence from the initial propagation node degree or from the initial propagation node attribute (K-shell value), it needs to be further discussed), the density curve of each node changes with time ahead of the time when the peak value appears in the numerical simulation, while the peak value also increased. The results obtained by quantifying the parameters quantified as a screening mechanism through simulation experiments and numerical simulations were different, which may be related to the topological structure of network not considered in the numerical simulation.

Each individual node may have different interests in different types of public opinion because of their own cultural heritage, knowledge, social experience, etc. The different physical networks and changing human psychology in which the individual is located also have a great impact on the spread of public opinion. Therefore, it is very difficult to use a single model and simulation method to obtain all public opinion dissemination results very accurately. However, through in-depth research and continuous development of our understanding of the real world, mathematical models based on mathematical abstraction methods can better characterize the spread of public opinion in reality [32-36]. In this paper, we have established a public opinion dissemination SEIR model with a screening mechanism and achieved some interesting results. But there is still room for improvement in our research process and results. For example, we can further study how the screening effect changes over time and how each parameter in the propagation model changes over time. Moreover, in the process of simulation experiment, we can further analyze the propagation influence of initial dissemination node attribute (K-shell value).

There are also some meaningful topics to dig in, for example: (1) although the quantitative description formula of the screening mechanism is obtained, the change of parameters with time is not reflected in the simulation experiment. (2) The selected data set is single, and several groups of data sets should be selected for simulation experiments to compare and analyze the applicability of models on different types of networks. (3) If the real public opinion communication events are selected as the case study, the accuracy of the model prediction will be more persuasive [37].

\section{Data Availability}

Some or all data, models, or code that support the findings of this study are available from the corresponding author upon reasonable request.

\section{Conflicts of Interest}

The authors declare that they have no conflicts of interest. 


\section{Acknowledgments}

This paper was supported by the MOE (Ministry of Education in China) Humanities and Social Sciences Foundation (grant no. 20YJC860005) and Research Program of Science and Technology at Universities of Inner Mongolia Autonomous Region (grant no. NJZZ20150).

\section{References}

[1] We Are Social, "Global digital report," 2018, http:// wearesocial.cn/blog/2018/01/30/2018-global-digital-report/.

[2] Howmet, "Subject based document retrieval from," 2020, https://www.cnki.net.

[3] W. O. Kermack and A. G. McKendrick, "A contribution to the mathematical theory of epidemics," Proceedings of the Royal Society Lond A, vol. 115, pp. 700-721, 1927.

[4] W. O. Kermack and A. G. McKendrick, "Contributions to the mathematical theory of epidemics. III. Further studies of the problem of endemicity," Proceedings of the Royal Society of London. Series A, Containing Papers of a Mathematical and Physical Character, vol. 141, pp. 94-122, 1933.

[5] J. D. Cui, Q. Zheng, and S. Sun, "Research on topic-based public opinion dissemination in micro blogging based on improved SEIR model," Information Science, vol. 35, no. 12, pp. 22-27, 2017.

[6] C. L. Huang, X. W. Liu, and M. H. Deng, "Over view of traceability algorithms for disease transmission on complex networks," Journal of Computer Science, vol. 41, no. 6, pp. 190-213, 2018.

[7] S. C. Han, Y. Liu, and Y. C. Zhang, "The herding effect of public opinion dissemination based on dynamic evolutionary game theory," Journal of Systems Engineering, vol. 26, no. 2, pp. 275-281, 2011.

[8] J. Liu and T. Zhang, "Epidemic spreading of an SEIRS model in scale-free networks," Communications in Nonlinear Science and Numerical Simulation, vol. 16, no. 8, pp. 3375-3384, 2011.

[9] Q. Liu, T. Li, and M. Sun, "The analysis of anSEIRrumor propagation model on heterogeneous network," Physica A: Statistical Mechanics and Its Applications, vol. 469, pp. 372380, 2017.

[10] Y. L. Ma, W. X. Xu, and Z. H. Zhang, "Stability analysis of SEIR model with general contact rate," Journal of China University of Science and Technology, vol. 45, no. 9, pp. 737-744, 2015.

[11] Q. E. Ma and J. Zhang, "Research on the mechanism of Wechat public signal transmission on complex networks based on SIR model," Information Science, vol. 36, no. 7, pp. 32-37, 2018.

[12] C. Su, J. Peng, and S. G. Li, "Research on network public opinion propagation model based on uncertain differential equation," Systems Engineering Theory and Practice, vol. 35, no. 12, pp. 3201-3209, 2015.

[13] M. S. Shang and X. G. Qiu, "Integrated study of communication dynamics on social networks," Systems Engineering Theory and Practice, vol. 35, no. 10, pp. 2557-2563, 2015.

[14] X. L. Wang, L. J. Zhao, and W. L. Xie, "Rumor propagation model of forgetting rate change in scale-free networks," Systems Engineering Theory and Practice, vol. 35, no. 2, pp. 458-465, 2015.

[15] C. Wang, Y. P. Hu, C. Y. Liu, Z. Liu, and J. F. Ma, "Stability analysis of information spreading on SNS based on refined SEIR mode," China Communications, vol. 11, no. 11, pp. 24-33, 2014.
[16] D. I. Xu and X. P. Dai, "Computational experimental study of network public opinion dissemination under dynamic influence," Systems Engineering Theory and Practice, vol. 36, no. 12, pp. 3198-3209, 2016.

[17] C. Y. Xia, JH. Ma, and Q. Chen, "Study on SIR propagation model considering infection vectors on complex networks," Journal of Systems Engineering, vol. 25, no. 6, pp. 818-823, 2010.

[18] H. F. Zhao, Y. Q. Sun, and E. Tse, "Emergency public opinion dissemination based on bounded trust model," Systems Engineering Theory and Practice, vol. 37, no. 12, pp. 3244-3252, 2017.

[19] Y. Zan, "DSIR double-rumors spreading model in complex networks," Chaos, Solitons \& Fractals, vol. 110, pp. 191-202, 2018.

[20] S. Chen, "Research on public opinion communication in two - tier social network based on SEIR," Information Research, vol. 275, no. 9, pp. 29-36, 2020.

[21] L. J. Yin, S. M. Qi, L. W. Yu, and Y. R. Xiao, "An improved SEIR model for the emergency propagation in Universities," Journal of Mathematics in Practice and Theory, vol. 48, no. 22, pp. 107-113, 2018.

[22] C. Fan, H. Song, and G. Ding, "Research on an improved SEIR network rumor propagation model," Journal of Intelligence, vol. 36, no. 3, pp. 86-91, 2017.

[23] W. Q. T. Zhu, P. W. Zhao, and Q. X. Chun, "A novel O2O teaching model using mobile social network APP to combine online and offline teaching," in Proceedings of the 2016 8th International Conference on Information Technology in Medicine and Education (ITME), vol. 34, no. 6, pp. 92-97, Fuzhou, China, December 2016.

[24] Q. Li, Y. J. Du, Z. Y. Li et al., "HK-SEIR model of public opinion evolution based on communication factors," Engineering Applications of Artificial Intelligence, vol. 100, no. 2, Article ID 104192, 2021.

[25] D. Zhang, B. Sheng, and G. Sun, Modeling and Simulation of Network Public Opinion Propagation Model Based on Interest Matching in Social Network, Springer, Cham, Switzerland, Article ID 11633, 2019.

[26] G. Jiang, S. Li, and M. Li, "Dynamic rumor spreading of public opinion reversal on Weibo based on a two-stage SPNR model," Physica A: Statistical Mechanics and Its Applications, vol. 558, 2020.

[27] J. Q. Kan, J. R. Xie, and H. F. Zhang, "Impacts of social reinforcement and edge weight on the spreading of information in networks," Journal of University of Electronic Science and Technology of China, vol. 43, no. 1, pp. 21-25, 2014.

[28] R. A. Rossi and N. K. Ahmed, "The network data repository with interactive graph analytics and visualization," in Proceedings of the Twenty-Ninth AAAI Conference on Artificial Intelligence, AAAI Press, Austin, TX, USA, January 2015.

[29] W. X. Jin, B. Song, and G. Z. Liu, "Interdependent network vulnerability analysis based on correlation distribution function," Complex Systems and Complexity Science, vol. 013, no. 4, pp. 8-17, 2016.

[30] D. Wang and X. Y. Jin, "Modeling of scaleless networks with adjustable clustering coefficients and congestion problems," Journal of Physics, vol. 61, no. 22, pp. 543-551, 2012.

[31] M. Kitsak, L. K. Gallos, S. Havlin et al., "Identification of influential spreaders in complex networks," Nature Physics, vol. 6, no. 11, pp. 888-893, 2010.

[32] D. Brockmann and D. Helbing, "The hidden geometry of complex, network-driven contagion phenomena," Science, vol. 342, no. 6164, pp. 1337-1342, 2013. 
[33] E. X. Dejesus and C. Kaufman, "Routh-Hurwitz criterion in the examination of eigenvalues of a system of nonlinear ordinary differential equations," Physical Review A, vol. 35, no. 12 , pp. $5288-5290,1987$.

[34] N. Difonzo, N. M. Robinson, J. M. Suls, and C. Rini, "Rumors about cancer: content, sources, coping, transmission, and belief," Journal of Health Communication, vol. 17, no. 9, pp. 1099-1115, 2012.

[35] S. Devavrat and Z. Tauhid, "Rumors in a network: who's the culprit," IEEE Transactions on Information Theory, vol. 57, no. 8, pp. 5163-5181, 2011.

[36] E. Seo, P. Mohapatra, and T. Abdelzaher, "Identifying rumors and their sources in social networks," Proceedings of SPIE The International Society for Optical Engineering, vol. 8389, pp. 1-13, 2012.

[37] G. Wang, Y. Wang, K. Liu, and J. Li, "Multidimensional influencing factors of public opinion information dissemination in social media: evidence from Weibo dataset," International Journal of Modern Physics B, vol. 33, no. 31, Article ID 1950375, 2019. 